\title{
Insight Into Lightning Initiation via Downward Terrestrial Gamma-ray Flash Observations at Telescope Array
}

\author{
Jackson Remington ${ }^{a, *}$ on behalf of the Telescope Array Collaboration ${ }^{\dagger}$ \\ ${ }^{a}$ University of Utah, \\ 201 President's Cir., Salt Lake City, USA \\ E-mail: jremington@cosmic.utah.edu
}

Due to the difficulty of direct measurement of the thunderstorm environment, in particular the electric field strengths, the initial stages of lightning breakdown remain mysterious. The 1994 discovery of Terrestrial Gamma-ray Flashes (TGFs) and their implications for megaVolt potentials within thunderclouds has proved to be a valuable source of information about the breakdown process.

The Telescope Array Surface Detector (TASD) - a $700 \mathrm{~km}^{2}$ scintillator array in Western Utah, U.S.A - coupled with a lightning mapping array, fast sferic (field change) sensor and broadband interferometer, has provided unique insight into the properties of this energetic radiation and of lightning initiation in general. In particular, microsecond-scale timing comparisons have clearly established that downward TGFs occur during strong initial breakdown pulses (IBPs) of downward negative cloud-to-ground and intracloud flashes. In turn, the IBPs are produced by streamer-based fast negative breakdown.

Investigations into downward TGFs with the TASD have significantly evolved with recent upgrades to lightning instrumentation. A second state-of-the-art broadband interferometer allows high-resolution stereo observation of lightning development. A high-speed optical video camera, set to be deployed in Spring 2021, will allow simultaneous observation of the visual component of lightning responsible for TGF production. Finally, a suite of ground based static electric field mills will provide new information on the large-scale properties of the thunderstorms in which downward TGFs arise.

In this talk, we present the most recent TGF observations from the Telescope Array.

$3^{\text {th }}$ International Cosmic Ray Conference (ICRC 2021)

July 12th - 23rd, 2021

Online - Berlin, Germany

\footnotetext{
*Presenter

${ }^{\dagger}$ A complete list of authors can be found at the end of the proceedings
} 


\section{Introduction}

Terrestrial gamma-ray flashes (TGFs) were first discovered by the Compton Gamma-ray Observatory [1] and studied extensively from above by other satellites. After their correlation to lightning discharges, it was suggested that TGFs should also be produced downward due to the symmetrical charge structure within thunderstorms. The observation of "burst" events at Telescope Array (TA) [2] in association with lightning flashes opened the door for the study of energetic radiation from lightning using cosmic ray detectors. The installation of a VHF Lightning Mapping Array (LMA) and electric field sensors in 2014 confirmed that these events were indeed downward TGFs initiated in the first couple milliseconds of negative lightning flashes [3]. A recent upgrade in 2018 added a broadband interferometer (INTF), making TA the most robust site for the high-resolution study of downward TGFs.

All lightning breakdown begins with leader propagation, wherein short $\sim 50 \mathrm{~m}$ segments of air are ionized discretely between charge regions within a cloud or between a cloud and the ground below. Once a channel of air between these regions has heated to a conducting state, current flows extremely quickly, resulting in a bright lightning stroke. Somewhere in this process during rare, strong flashes, huge amounts of gamma rays can be emitted as TGFs, though the detailed process remains rather mysterious. A common view of TGF production involves the development of electromagnetic showers in the ambient electric fields of thunderstorms. When this occurs with sufficient field strength, the result is a relativistic runaway electron avalanche (RREA) [4], wherein electrons gain more energy from acceleration than they lose via atmospheric interactions (Figure 1). These showers produce large amounts of bremsstrahlung radiation, but require seed electrons having energy above $200 \mathrm{keV}$. One theory is that seed electrons are produced as secondary particles in cosmic-ray extensive air showers [5]. Another is that of thermal runaway, where strong, local electric fields excite electrons from a thermal state into the relativistic regime [6].

\section{Observations}

Four TGFs were observed in late 2018 [7] after the interferometer's installation, three of which (TGFs A, B, and C) were negative cloud-to-ground strokes occurring in the same storm on August 2, and the fourth (TGF D) being a negative intracloud flash on October 3. Each of these flashes began with strong initial breakdown pulses (IBPs), producing strong, impulsive signals (sferics) in the electric field change data. Breakdown activity was also mapped in three dimensions by the LMA and in two-dimensions by the higher-resolution interferometer (see Figure 2). The flashes of TGFs A, B, and C registered as having peak currents of $-38.3,-26.5$, and $-26.8 \mathrm{kA}$ by the National Lightning Detection Network (NLDN). TGF D did not trigger a measurement.

Surface detectors (TASDs) underneath the flashes triggered events at nearly the same time as these flashes - two consecutive bursts were detected in each of the parent flashes of TGFs A, C, and D and only a single burst for that of TGF B. The left-hand panels of Figure 3 shows the footprint of active detectors for both triggers of TGF C covering $\sim 5$ and $10 \mathrm{~km}^{2}$, respectively. The lowerright panel gives the detector response of the TASD with most energy deposit, displaying a very different profile to that of a cosmic ray air shower. The jagged and inconsistent response between scintillator layers is indicative of a developed electromagnetic shower dominated by photons. 


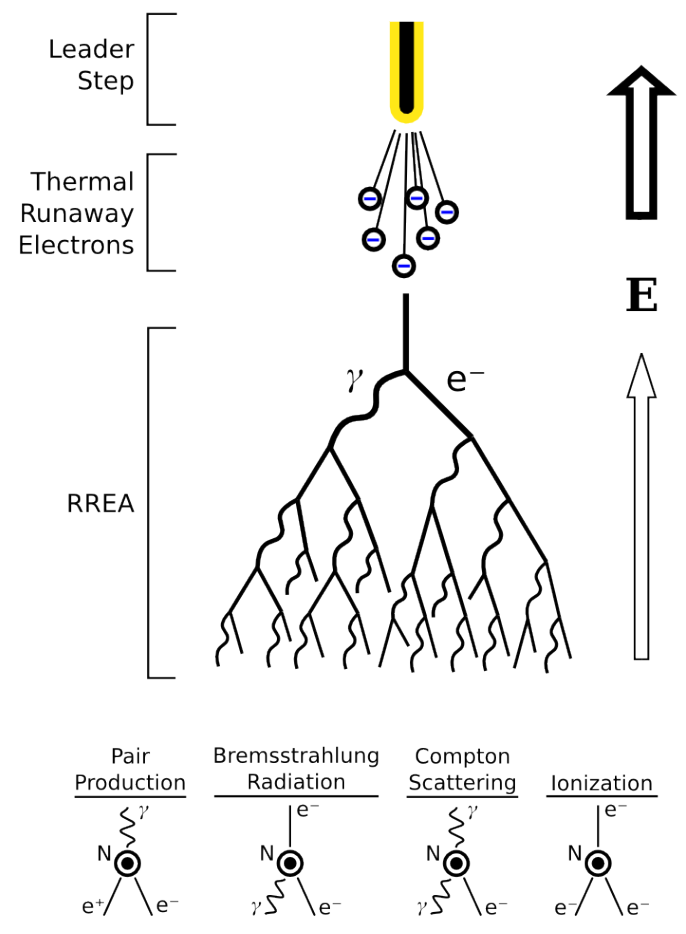

Figure 1: Illustration of the thermal runaway model of RREA development. High charge concentration in leader tips creates very strong, local electric fields. If sufficiently strong, thermal electrons can be accelerated to hundreds of $\mathrm{keV}$ or more. These energetic electrons can subsequently run away in the ambient thunderstorm electric fields to produce TGFs in the form of RREA.

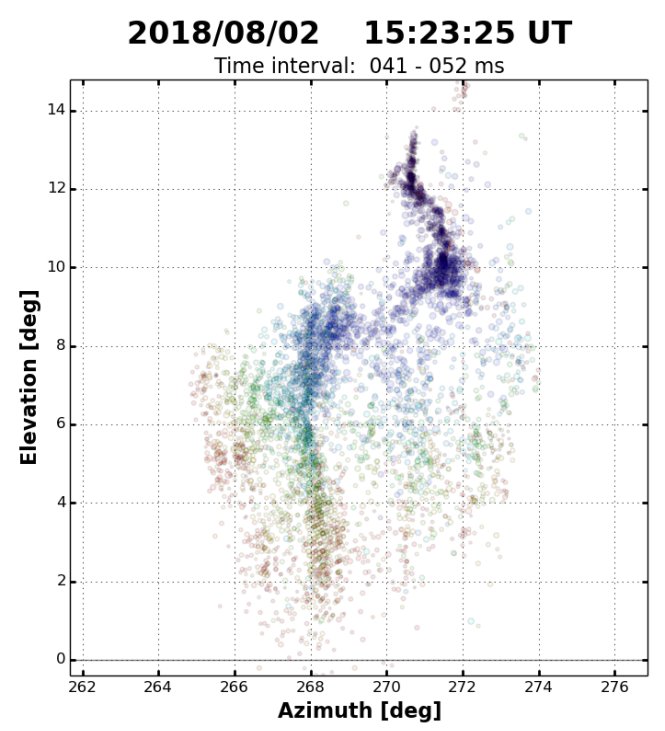

Figure 2: INTF data of TGF B showing the downward development of its parent flash. Points are colored from blue to red with respect to time and with sizes corresponding to power. 

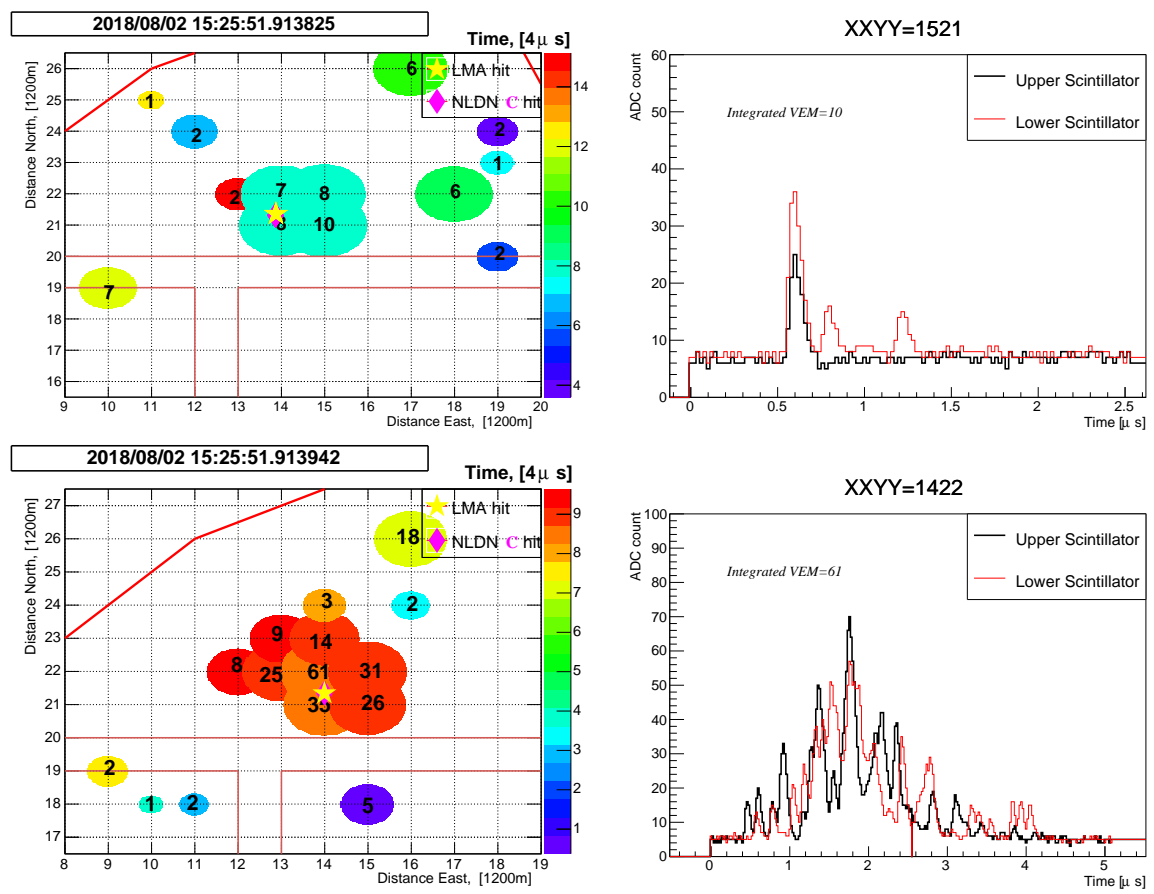

Figure 3: Footprints for the two triggers of TGF C and the responses of individual TASDs from the burst. Numbers in footprint circles indicate the detectors' total energy deposits in VEM while colors represent relative onset times in units of $4 \mu \mathrm{s}$. The yellow star indicates the median plan location of LMA sources. The purple diamond indicates the associated NLDN event, but is difficult to see due to being closely co-located with the LMA median point. The signal of TASD 1521 (top right) during the first gamma burst illustrates the fact that SDs are capable of distinguishing individual Compton electrons in low-fluence detections.

\section{Analysis}

Because the TASDs and lightning detectors measure different phenomena and were not designed to work together, special care is taken to correlate their results in a meaningful way. For example, the INTF carries no information about the TGF itself while the TASDs carry no information about the development of lightning breakdown at the time of TGF production. In addition, TA's large size of $\sim 700 \mathrm{~km}^{2}$ paired with the INTF's offset location means that propagation times between VHF lightning flash data and a downward-directed TGF can be as high as $100 \mu$ s (Figure 4). In order to examine discharge properties during TGF production on the order of a microsecond, numerical methods are used to correct for the different observed reference frames.

Two methods were developed independently for this purpose. The first iterates through possible TGF source times and locations to zero-in on a solution which best represents the data collected by all instruments. The second steps through individual INTF data points and calculates the expected time of TGF arrival at each TASD station, selecting a time and location which is most consistent with data. Both methods produced consistent results for all events, further verifying their validity. Figure 5 illustrates the solution of the stepping method for TGF A with an altitude of $3.2 \mathrm{~km}$ above ground. 


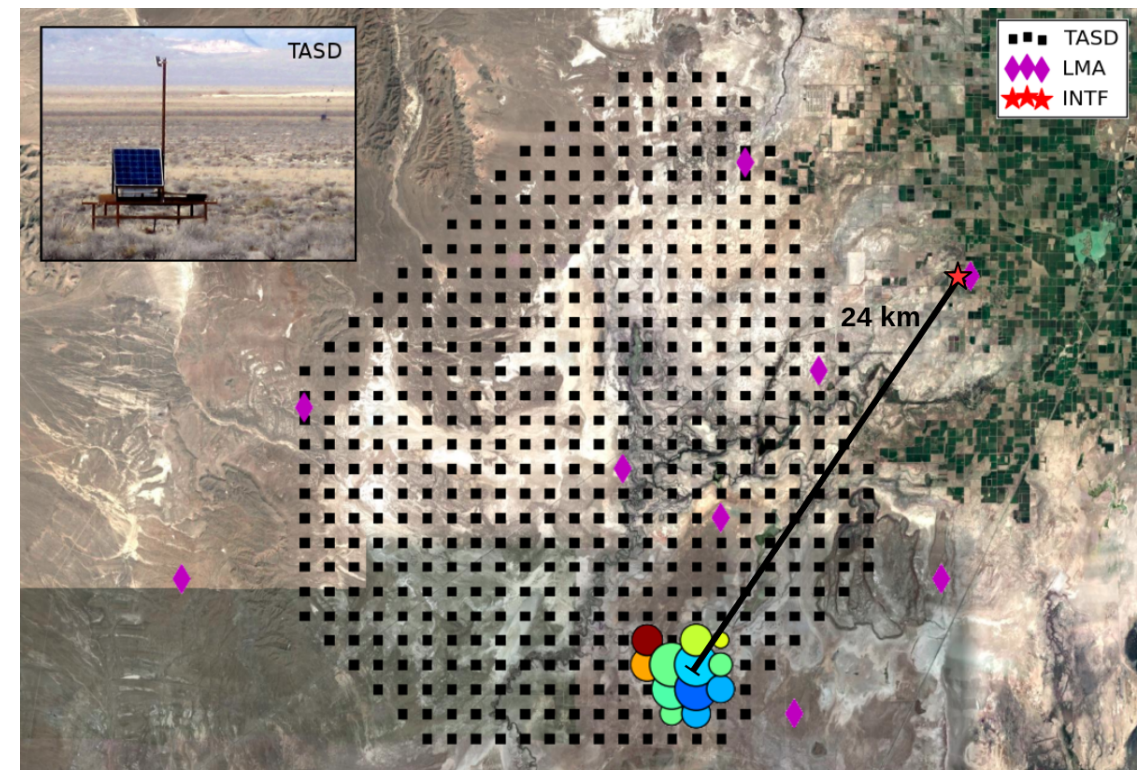

Figure 4: Map of the original TA during observation in 2018. TASDs are given as black squares, LMA stations are given as purple diamonds, and the INTF as the red star. The footprint of TGF D is overlayed on the array as an example with its distance from the INTF station. A photo of a surface detector is inlaid in the upper right.

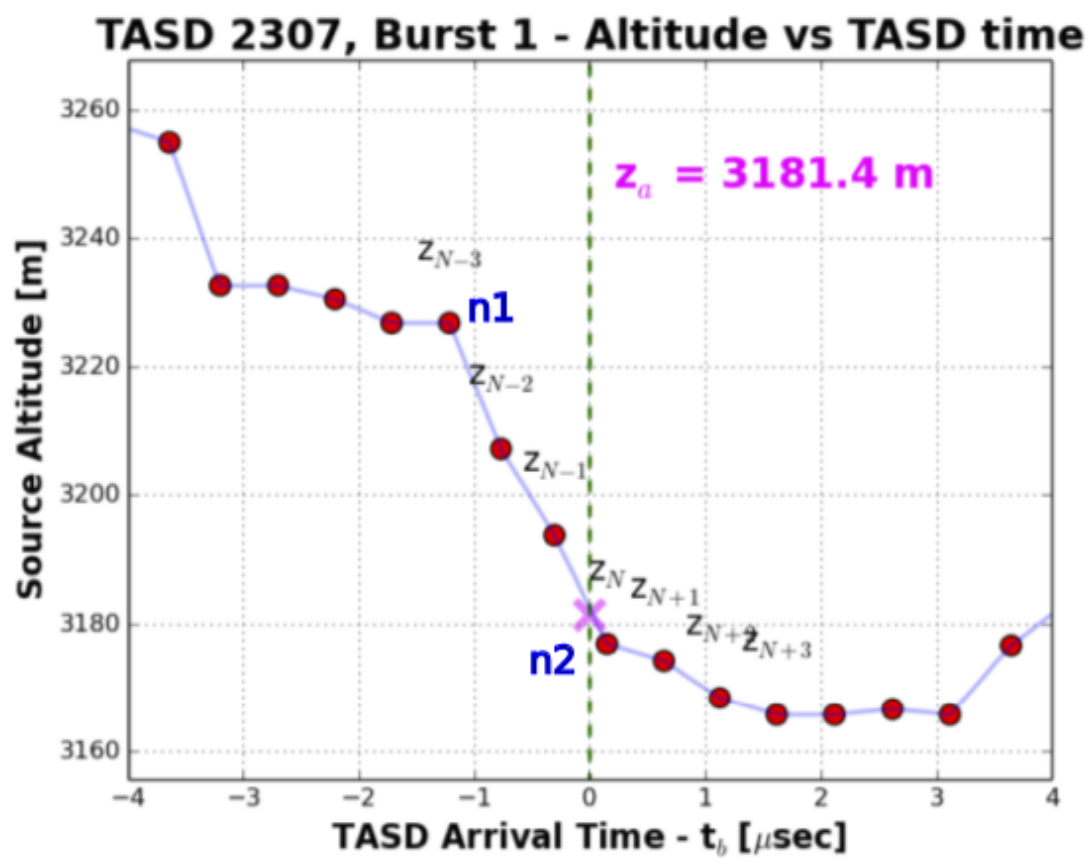

Figure 5: An illustration of the stepping method for TGF A. Each INTF point is examined at $0.5 \mu$ s intervals and taken as the TGF source time/location. The most representative solution is then interpolated from the two best-fitting points. 


\section{Results and Discussion}

As a result of the analyses outlined above, sources were identified for each TGF on the order of a microsecond or better. In terms of location, source uncertainties ranged from 40-300 m in the horizontal and 10-40 $\mathrm{m}$ in the vertical. Figure 6 shows the solution for TGF D as an example with TASD particle response data shifted to account for propagation effects. The TGF source is indicated by the horizontal and vertical black bars. These results show the first confirmation of downward TGFs and IBPs (green waveform). In fact, the strongest gamma burst of each TGF occurs during the flash's strongest IBP. Also note that the IBP is accompanied by an interval of increased power and speed in INTF points. this is the case for all IBPs of the TGFs, representing the individual leader steps discussed in section 1.

These periods of fast leader propagation reach speeds on the order of $10^{7} \mathrm{~m} / \mathrm{s}$, an order of magnitude faster than standard leader propagation which requires the air to heat up and ionize. This is indicative of a newly-discovered process called fast negative breakdown (FNB) [], consisting of negative streamer systems developing in the strong electric fields near leader tips. Although the statistics are low, the fact that all bursts occur during periods of FNB suggests that RREA is being seeded by thermal electrons accelerated to high energy by these streamer systems in local electric fields rather than by cosmic ray secondaries.

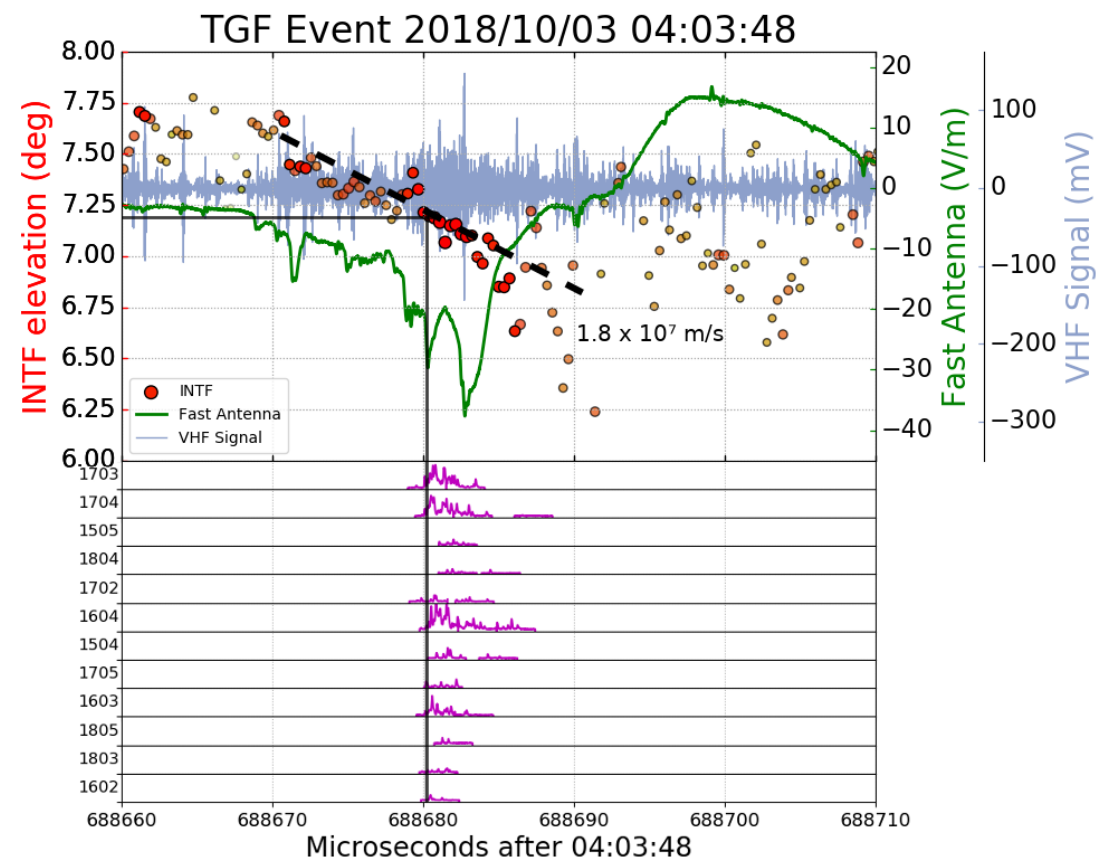

Figure 6: Source determination for TGF D. Circles indicate INTF points with color, size, and opacity representing power. Light blue waveform shows the raw VHF waveform. Green curve shows the electric field change recorded by the fast sferic sensor. Purple curves along lower axes are particle responses of all active surface detectors. Black vertical and horizontal bars show the solution of the TGF source resulting from the iterative analysis method. Note the TGF production is associated with the flash's largest IBP and with an interval of fast leader propagation indicating streamer-based fast negative breakdown. 


\section{Acknowledgements}

The Telescope Array experiment is supported by the Japan Society for the Promotion of Science(JSPS) through Grants-in-Aid for Priority Area 431, for Specially Promoted Research JP21000002, for Scientific Research (S) JP19104006, for Specially Promoted Research JP15H05693, for Scientific Research (S) JP15H05741 and JP19H05607, for Science Research (A) JP18H03705, for Young Scientists (A) JPH26707011, and for Fostering Joint International Research (B) JP19KK0074, by the joint research program of the Institute for Cosmic Ray Research (ICRR), The University of Tokyo; by the Pioneering Program of RIKEN for the Evolution of Matter in the Universe (r-EMU); by the U.S. National Science Foundation awards PHY-1404495, PHY-1404502, PHY-1607727, PHY-1712517, PHY-1806797 and PHY-2012934; by the National Research Foundation of Korea (2017K1A4A3015188, 2020R1A2C1008230, \& 2020R1A2C2102800) ; by the Ministry of Science and Higher Education of the Russian Federation under the contract 075-15-2020-778, RFBR grant 20-02-00625a (INR), IISN project No. 4.4501.18, and Belgian Science Policy under IUAP VII/37 (ULB). This work was partially supported by the grants of The joint research program of the Institute for Space-Earth Environmental Research, Nagoya University and Inter-University Research Program of the Institute for Cosmic Ray Research of University of Tokyo. The foundations of Dr. Ezekiel R. and Edna Wattis Dumke, Willard L. Eccles, and George S. and Dolores Doré Eccles all helped with generous donations. The State of Utah supported the project through its Economic Development Board, and the University of Utah through the Office of the Vice President for Research. The experimental site became available through the cooperation of the Utah School and Institutional Trust Lands Administration (SITLA), U.S. Bureau of Land Management (BLM), and the U.S. Air Force. We appreciate the assistance of the State of Utah and Fillmore offices of the BLM in crafting the Plan of Development for the site. Patrick A. Shea assisted the collaboration with valuable advice and supported the collaborationa ${ }^{\mathrm{TM}} \mathrm{S}$ efforts. The people and the officials of Millard County, Utah have been a source of steadfast and warm support for our work which we greatly appreciate. We are indebted to the Millard County Road Department for their efforts to maintain and clear the roads which get us to our sites. We gratefully acknowledge the contribution from the technical staffs of our home institutions. An allocation of computer time from the Center for High Performance Computing at the University of Utah is gratefully acknowledged.

\section{References}

[1] Fishman, G. J., et al. (1994). Discovery of Intense Gamma-Ray Flashes of Atmospheric Origin. American Association for the Advancement of Science, 264 (5163), 1313-1316. 10.1126/science.264.5163.1313

[2] Abbasi, R., et al. (2017). The Bursts of High Energy Events Observed by the Telescope Array Surface Detector. Physics Letters A, 381 (32), 2565-2572. 10.1016/j.physleta.2017.06.022

[3] Abbasi, R., et al. (2018). Gamma Ray Showers Observed at Ground Level in Coincidence With Downward Lightning Leaders. Journal of Geophysical Research: Atmospheres, 123 (13), 6864-6879. 10.1029/2017JD027931 
[4] Wilson, C. T. R. (1924). The Electric Field of a Thundercloud and Some of its Effects. Proceedings of the Physical Society of London, 37 (1), 32D-37D. 10.1088/1478-7814/37/1/314

[5] Gurevich, A. V., et al. (1992). Runaway electron mechanism of air breakdown and preconditioning during a thunderstorm Physics Letters A, 165 (5), 463-468. 10.1016/03759601(92)90348-P

[6] Moss, G., et al. (2006). Monte Carlo Model for Analysis of Thermal Runaway Electrons in Streamer Tips in Transient Luminous Events and Streamer Zones of Lightning Leaders. Journal of Geophysical Research, 111 (A02307). 10.1029/2005JA011350

[7] Belz, J. W., et al. (2020). Observations of the Origin of Downward Terrestrial Gamma-Ray Flashes. Journal of Geophysical Research: Atmospheres, 125 (23). 10.1029/2019JD031940

[8] Tilles, J., et al. (2019). Fast Negative Breakdown in Thunderstorms. Nature Communications, 10, 1648. 10.1038/s41467-019-09621-z 


\section{Telescope Array Collaboration Full Author List}

R.U. Abbasi ${ }^{1}$, M. Abe ${ }^{2}$, T. Abu-Zayyad ${ }^{1,3}$, M. Allen ${ }^{3}$, Y. Arai ${ }^{4}$, E. Barcikowski ${ }^{3}$, J.W. Belz ${ }^{3}$, D.R. Bergman ${ }^{3}$, S.A. Blake ${ }^{3}$, I. Buckland ${ }^{3}$, R. $\mathrm{Cady}^{3}$, B.G. Cheon ${ }^{5}$, J. Chiba ${ }^{6}$, M. Chikawa ${ }^{7}$, T. Fujii ${ }^{8}$, K. Fujisue ${ }^{7}$, K. Fujita ${ }^{4}$, R. Fujiwara ${ }^{4}$, M. Fukushima ${ }^{7,9}$, R. Fukushima ${ }^{4}$, G. Furlich ${ }^{3}$, R. Gonzalez ${ }^{3}$, W. Hanlon ${ }^{3}$, M. Hayashi ${ }^{10}$, N. Hayashida ${ }^{11}$, K. Hibino ${ }^{11}$, R. Higuchi ${ }^{7}$, K. Honda ${ }^{12}$, D. Ikeda ${ }^{11}$, T. Inadomi ${ }^{13}$, N. Inoue ${ }^{2}$, T. Ishii ${ }^{12}$, H. Ito ${ }^{14}$, D. Ivanov ${ }^{3}$, H. Iwakura ${ }^{13}$, H.M. Jeong ${ }^{15}$, S. Jeong ${ }^{15}$, C.C.H. Jui ${ }^{3}$, K. Kadota ${ }^{16}$, F. Kakimoto ${ }^{11}$, O. Kalashev ${ }^{17}$, K. Kasahara ${ }^{18}$, S. Kasami ${ }^{19}$, H. Kawai ${ }^{20}$, S. Kawakami ${ }^{4}$, S. Kawana ${ }^{2}$, K. Kawata ${ }^{7}$, E. Kido ${ }^{14}$, H.B. Kim ${ }^{5}$, J.H. Kim ${ }^{3}$, J.H. Kim ${ }^{3}$, M.H. Kim ${ }^{15}$, S.W. Kim ${ }^{15}$, Y. Kimura ${ }^{4}$, S. Kishigami ${ }^{4}$, Y. Kubota ${ }^{13}$, S. Kurisu ${ }^{13}$, V. Kuzmin ${ }^{17^{*}}$, M. Kuznetsov ${ }^{17,21}$, Y.J. Kwon ${ }^{22}$, K.H. Lee ${ }^{15}$, B. Lubsandorzhiev ${ }^{17}$, J.P. Lundquist ${ }^{3,23}$, K. Machida ${ }^{12}$, H. Matsumiya ${ }^{4}$, T. Matsuyama ${ }^{4}$, J.N. Matthews ${ }^{3}$, R. Mayta ${ }^{4}$, M. Minamino ${ }^{4}$, K. Mukai ${ }^{12}$, I. Myers ${ }^{3}$,

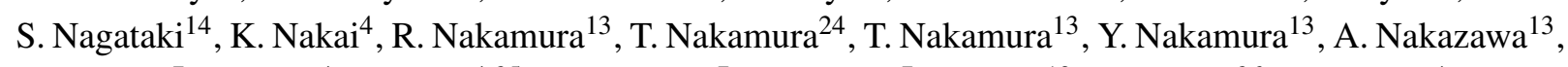
T. Nonaka ${ }^{7}$, H. Oda ${ }^{4}$, S. Ogio ${ }^{4,25}$, M. Ohnishi ${ }^{7}$, H. Ohoka ${ }^{7}$, Y. Oku ${ }^{19}$, T. Okuda ${ }^{26}$, Y. Omura ${ }^{4}$, M. Ono ${ }^{14}$, R. Onogi ${ }^{4}$, A. Oshima ${ }^{4}$, S. Ozawa ${ }^{27}$, I.H. Park ${ }^{15}$, M. Potts ${ }^{3}$, M.S. Pshirkov ${ }^{17,28}$, J. Remington ${ }^{3}$, D.C. Rodriguez ${ }^{3}$, G.I. Rubtsov ${ }^{17}$, D. Ryu ${ }^{29}$, H. Sagawa ${ }^{7}$, R. Sahara ${ }^{4}$, Y. Saito ${ }^{13}$, N. Sakaki ${ }^{7}$, T. Sako ${ }^{7}$, N. Sakurai ${ }^{4}$, K. Sano ${ }^{13}$, K. Sato ${ }^{4}$, T. Seki ${ }^{13}$, K. Sekino ${ }^{7}$, P.D. Shah ${ }^{3}$, Y. Shibasaki ${ }^{13}$, F. Shibata ${ }^{12}$, N. Shibata $^{19}$, T. Shibata ${ }^{7}$, H. Shimodaira ${ }^{7}$, B.K. Shin ${ }^{29}$, H.S. Shin ${ }^{7}$, D. Shinto ${ }^{19}$, J.D. Smith ${ }^{3}$, P. Sokolsky ${ }^{3}$, N. Sone ${ }^{13}$, B.T. Stokes ${ }^{3}$, T.A. Stroman ${ }^{3}$, T. Suzawa ${ }^{2}$, Y. Takagi ${ }^{4}$, Y. Takahashi ${ }^{4}$, M. Takamura ${ }^{6}$, M. Takeda ${ }^{7}$, R. Takeishi ${ }^{7}$, A. Taketa $^{30}$, M. Takita ${ }^{7}$, Y. Tameda ${ }^{19}$, H. Tanaka ${ }^{4}$, K. Tanaka ${ }^{31}$, M. Tanaka ${ }^{32}$, Y. Tanoue ${ }^{4}$, S.B. Thomas ${ }^{3}$, G.B. Thomson ${ }^{3}$, P. Tinyakov ${ }^{17,21}$, I. Tkachev ${ }^{17}$, H. Tokuno ${ }^{33}$, T. Tomida ${ }^{13}$, S. Troitsky ${ }^{17}$, R. Tsuda ${ }^{4}$, Y. Tsunesada ${ }^{4,25}$, Y. Uchihori ${ }^{34}$, S. Udo $^{11}$,

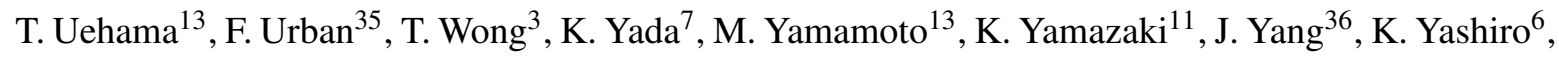
F. Yoshida ${ }^{19}$, Y. Yoshioka ${ }^{13}$, Y. Zhezher ${ }^{7,17}$, and Z. Zundel ${ }^{3}$

${ }^{1}$ Department of Physics, Loyola University Chicago, Chicago, Illinois, USA

2 The Graduate School of Science and Engineering, Saitama University, Saitama, Saitama, Japan

${ }^{3}$ High Energy Astrophysics Institute and Department of Physics and Astronomy, University of Utah, Salt Lake City, Utah, USA

${ }^{4}$ Graduate School of Science, Osaka City University, Osaka, Osaka, Japan

${ }^{5}$ Department of Physics and The Research Institute of Natural Science, Hanyang University, Seongdong-gu, Seoul, Korea

${ }^{6}$ Department of Physics, Tokyo University of Science, Noda, Chiba, Japan

${ }^{7}$ Institute for Cosmic Ray Research, University of Tokyo, Kashiwa, Chiba, Japan

${ }^{8}$ The Hakubi Center for Advanced Research and Graduate School of Science, Kyoto University, Kitashirakawa-Oiwakecho, Sakyo-ku, Kyoto, Japan

${ }^{9}$ Kavli Institute for the Physics and Mathematics of the Universe (WPI), Todai Institutes for Advanced Study, University of Tokyo, Kashiwa, Chiba, Japan

${ }^{10}$ Information Engineering Graduate School of Science and Technology, Shinshu University, Nagano, Nagano, Japan

${ }^{11}$ Faculty of Engineering, Kanagawa University, Yokohama, Kanagawa, Japan

${ }^{12}$ Interdisciplinary Graduate School of Medicine and Engineering, University of Yamanashi, Kofu, Yamanashi, Japan

${ }^{13}$ Academic Assembly School of Science and Technology Institute of Engineering, Shinshu University, Nagano, Nagano, Japan

${ }^{14}$ Astrophysical Big Bang Laboratory, RIKEN, Wako, Saitama, Japan

15 Department of Physics, Sungkyunkwan University, Jang-an-gu, Suwon, Korea

16 Department of Physics, Tokyo City University, Setagaya-ku, Tokyo, Japan

${ }^{17}$ Institute for Nuclear Research of the Russian Academy of Sciences, Moscow, Russia

${ }^{18}$ Faculty of Systems Engineering and Science, Shibaura Institute of Technology, Minato-ku, Tokyo, Japan 
${ }^{19}$ Department of Engineering Science, Faculty of Engineering, Osaka Electro-Communication University, Neyagawashi, Osaka, Japan

${ }^{20}$ Department of Physics, Chiba University, Chiba, Chiba, Japan

${ }^{21}$ Service de Physique ThÃ@)orique, UniversitÃ C Libre de Bruxelles, Brussels, Belgium

${ }^{22}$ Department of Physics, Yonsei University, Seodaemun-gu, Seoul, Korea

${ }^{23}$ Center for Astrophysics and Cosmology, University of Nova Gorica, Nova Gorica, Slovenia

${ }^{24}$ Faculty of Science, Kochi University, Kochi, Kochi, Japan

${ }^{25}$ Nambu Yoichiro Institute of Theoretical and Experimental Physics, Osaka City University, Osaka, Osaka, Japan

${ }^{26}$ Department of Physical Sciences, Ritsumeikan University, Kusatsu, Shiga, Japan

${ }^{27}$ Quantum ICT Advanced Development Center, National Institute for Information and Communications Technology, Koganei, Tokyo, Japan

${ }^{28}$ Sternberg Astronomical Institute, Moscow M.V. Lomonosov State University, Moscow, Russia

${ }^{29}$ Department of Physics, School of Natural Sciences, Ulsan National Institute of Science and Technology, UNIST-gil, Ulsan, Korea

${ }^{30}$ Earthquake Research Institute, University of Tokyo, Bunkyo-ku, Tokyo, Japan

31 Graduate School of Information Sciences, Hiroshima City University, Hiroshima, Hiroshima, Japan

32 Institute of Particle and Nuclear Studies, KEK, Tsukuba, Ibaraki, Japan

${ }^{33}$ Graduate School of Science and Engineering, Tokyo Institute of Technology, Meguro, Tokyo, Japan

${ }^{34}$ Department of Research Planning and Promotion, Quantum Medical Science Directorate, National Institutes for Quantum and Radiological Science and Technology, Chiba, Chiba, Japan

${ }^{35}$ CEICO, Institute of Physics, Czech Academy of Sciences, Prague, Czech Republic

${ }^{36}$ Department of Physics and Institute for the Early Universe, Ewha Womans University, Seodaaemun-gu, Seoul, Korea

${ }^{*}$ Deceased 